\title{
Leg Skin
}

National Cancer Institute

\section{Source}

National Cancer Institute. Leg Skin. NCI Thesaurus. Code C52749.

The skin or integument surrounding the leg. 